\title{
Autophagy inhibition radiosensitizes in vitro, yet reduces radioresponses in vivo due to deficient immunogenic signalling
}

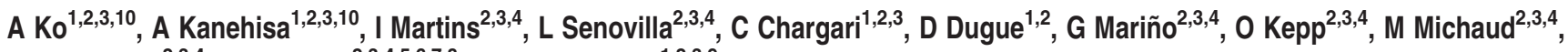 \\ J-L Perfettini ${ }^{2,3,4}$, G Kroemer $^{2,3,4,5,6,7,8}$ and E Deutsch ${ }^{*, 1,2,3,9}$
}

\begin{abstract}
Clinical oncology heavily relies on the use of radiotherapy, which often leads to merely transient responses that are followed by local or distant relapse. The molecular mechanisms explaining radioresistance are largely elusive. Here, we identified a dual role of autophagy in the response of cancer cells to ionizing radiation. On one hand, we observed that the depletion of essential autophagy-relevant gene products, such as ATG5 and Beclin 1, increased the sensitivity of human or mouse cancer cell lines to irradiation, both in vitro (where autophagy inhibition increased radiation-induced cell death and decreased clonogenic survival) and in vivo, after transplantation of the cell lines into immunodeficient mice (where autophagy inhibition potentiated the tumour growth-inhibitory effect of radiotherapy). On the other hand, when tumour proficient or deficient for autophagy were implanted in immunocompetent mice, it turned out that defective autophagy reduced the efficacy of radiotherapy. Indeed, radiotherapy elicited an anti-cancer immune response that was dependent on autophagy-induced ATP release from stressed or dying tumour cells and was characterized by dense lymphocyte infiltration of the tumour bed. Intratumoural injection of an ecto-ATPase inhibitor restored the immune infiltration of autophagy-deficient tumours post radiotherapy and improved the growth-inhibitory effect of ionizing irradiation. Altogether, our results reveal that beyond its cytoprotective function, autophagy confers immunogenic properties to tumours, hence amplifying the efficacy of radiotherapy in an immunocompetent context. This has far-reaching implications for the development of pharmacological radiosensitizers.
\end{abstract}

Cell Death and Differentiation (2014) 21, 92-99; doi:10.1038/cdd.2013.124; published online 13 September 2013

Radiation is a widely used therapeutic approach for the loco-regional management of numerous cancerous malignancies. Depending on the location and tumour stage, radiation therapy can be administered alone or in combination with surgery, chemotherapy and/or hormonal treatments. In response to irradiation, tumour cells can die through different mechanisms such as apoptosis, necrosis and mitotic catastrophe, ${ }^{1,2}$ which in many cases are preceded or accompanied by pro-survival processes such as autophagy. ${ }^{3}$ The type of cellular response(s) and its relative contribution to the anti-tumour effect depends on several factors. In fact, intrinsic parameters such as the tumour cellular type, the cell cycle phase in which the irradiated cells are, as well as treatment protocol parameters, such as radiation dose and treatment intervals, may strongly influence the tumour response to irradiation. ${ }^{4}$
Macroautophagy (herein referred to as 'autophagy') involves the highly regulated sequestration of cytoplasmic organelles or bulk portions of cytosol inside two-membraned vesicules, named autophagosomes, which eventually fuse with lysosomes for the degradation of their inner autophagosome membrane and luminal content. ${ }^{5}$ Autophagy has an essential role in cellular adaptation to multiple types of stress, recycling of superfluous or damaged cellular material, quality control of organelles, removal of protein aggregates and destruction of intracellular pathogens., ${ }^{6,7}$ The discovery of autophagy-related genes (ATG) in the yeast Saccharomyces cerevisiae (reviewed in Klionsky et $a l^{8}$ ) has facilitated the molecular dissection of autophagy and participated to the better understanding of pathophysiological role of autophagy, including the generation, progression and therapeutic response of cancers. ${ }^{9}$ The formation of the autophagosome

\footnotetext{
${ }^{1}$ INSERM U1030, Radiothérapie moléculaire SIRIC SOCRATES, LABEX LERMIT \& DHU TORINO, Institut Gustave Roussy, Villejuif, France; ${ }^{2}$ Gustave Roussy Cancer Campus, Villejuif, France; ${ }^{3}$ SIRIC SOCRATES, LABEX LERMIT \& DHU TORINO, Université Paris Sud-Paris 11, Villejuif, France; ${ }^{4}$ INSERM U848, Institut Gustave Roussy, Villejuif, France; ${ }^{5}$ Metabolomics and Cell Biology Platforms, Institut Gustave Roussy, Villejuif, France; ${ }^{6}$ Equipe 11 labellisée Ligue contre le Cancer, Centre de Recherche des Cordeliers, Paris, France; ${ }^{7}$ Pôle de Biologie, Hôpital Européen Georges Pompidou, AP-HP, Paris, France; ${ }^{8}$ Université Paris Descartes, Paris 5, Paris, France and ${ }^{9}$ Department of Radiation Oncology, Institut Gustave Roussy, Villejuif, France

*Corresponding author: E Deutsch, INSERM U1030, Institut Gustave Roussy, Pavillon de Recherche 1, 39 rue Camille Desmoulins, F-94805 Villejuif, France. Tel: +33 1421152 96; Fax: +331421152 36; E-mail: eric.deutsch@gustaveroussy.fr

${ }^{10}$ These authors contributed equally to this work.

Keywords: radiotherapy; irradiation; autophagy; non-small-cell lung carcinoma; immunogenic cell death

Abbreviations: AGD, absolute growth delay; ATG, autophagy-related gene; ATP, adenosine triphosphate; BECN-1, beclin-1; CRT, calreticulin; DC, dendritic cell; $\mathrm{GAPDH}$, glyceraldehyde 3-phosphate dehydrogenase; HMGB1, high-mobility group 1; IR, ionizing radiation; LC3, microtubule-associated protein 1A/1B light chain 3; MAP, mitogen-activated protein; PE, phosphatidylethanolamine; PI3K, phosphatidylinositol 3-phosphate kinase; SCR, scramble; SF, surviving fraction; shRNA, short hairpin interfering RNA; siRNA, small interfering RNA; WT, wild type

Received 24.2.13; revised 03.7.13; accepted 10.7.13; Edited by D Rubinsztein; published online 13.9.13
} 
is based on subsequent protein conjugation steps. ${ }^{10}$ The initial formation of ATG5-ATG12 complexes allows for the recruitment of ATG8 (LC3 in mammalian cells) by phosphatidylethanolamine (PE). ATG5-ATG12 complexes are recycled to the cytosol before the complete formation of the autophagosome. ATG6 (Beclin 1 in mammalian cells) interacts with and activates phosphatidylinositol 3-phosphate kinase type III, thus generating phosphatidylinositol 3-phosphate-enriched membranes, which contribute to the recruitment of downstream autophagic factors. ${ }^{11}$ In tumour cells, autophagy acts as a double-edged sword. On one hand, autophagy is a haploinsufficient tumour suppressor mechanism, which promotes cancer cell proliferation and tumourigenesis, but on the other hand, autophagy favours cellular survival in low-oxygen/nutrient conditions or in response to toxic insults such as chemotherapeutic drugs or ionizing radiation (IR). ${ }^{12,13}$

Various preclinical models revealed that autophagy is activated in irradiated tumour cells ${ }^{14-16}$ and that ATG expression patterns are upregulated following irradiation. ${ }^{15}$ However, the role of autophagy in the resistance of cancer cells to radiation therapy remains controversial. Preclinical studies indicated that the sensitivity of tumour cells to radiation therapy could be enhanced by co-treatment with autophagy inducers, such as rapamycin, both in vitro and in lung cancer xenograft models. ${ }^{17}$ Consistently, treatment with autophagy inhibitors, such as 3-methyladenine, is associated with reduced sensitivity to radiation in models of advanced papillary thyroid cancer. ${ }^{18}$ In sharp contrast, autophagy induction contributes to radiation resistance of
$\mathrm{CD} 133^{+}$tumour cells, suggesting that autophagy inhibitors could be employed to radiosensitize cancer cells. ${ }^{19}$

In this context, we decided to (re-)evaluate the role of autophagy during tumour irradiation. Here, we report that irradiation-induced autophagy exerts a dual activity on tumour cell death and on tumour clearance by the immune system. On one hand, autophagy increases tumour cell survival by inhibiting cell death induction. However, on the other hand, autophagy contributes to the release of cell death-associated danger signals that trigger anti-tumour host immune responses.

\section{Results}

Autophagy inhibition reduces clonogenic survival after IR. Autophagy has previously been associated with either cell survival or cell death promoting mechanisms. Thus, we decided to determine the role of autophagy during IR. To this aim, we analysed the effect of ATG5 or Beclin 1 depletion in H460 and A549 cells. We also analysed the effect of ATG5 depletion in CT26 cells. Using short hairpin RNA (shRNA) we silenced the expression of ATG5 in A549, H460 and CT26 cells, and observed that following IR with 2 or $4 \mathrm{~Gy}$, the survival fraction (SF) determined by clonogenic assays was significantly reduced, as compared with cells that express unrelated shRNA controls (SCR) and thus had normal levels of autophagy. Thus, the inhibition of autophagy sensitizes A549, H460 and CT26 cells to IR-induced cell death (Figures $1 \mathrm{~b}-\mathrm{d}$ ). Following the same experimental
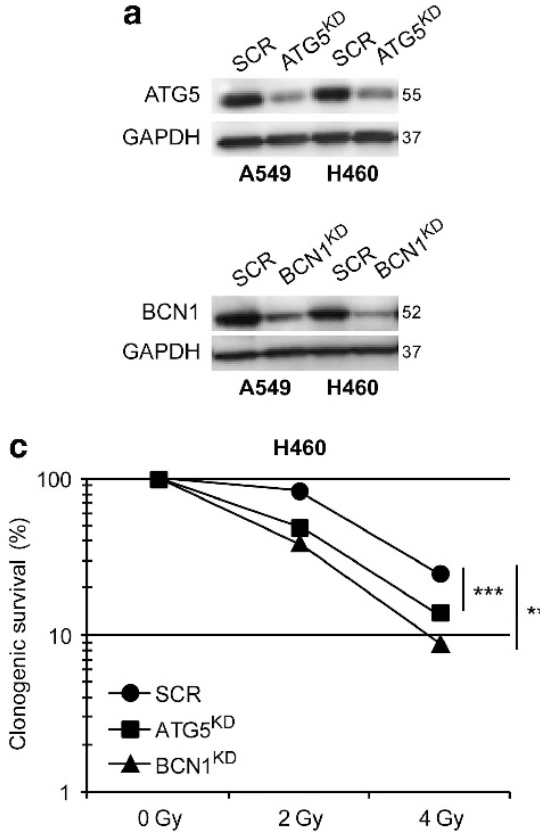
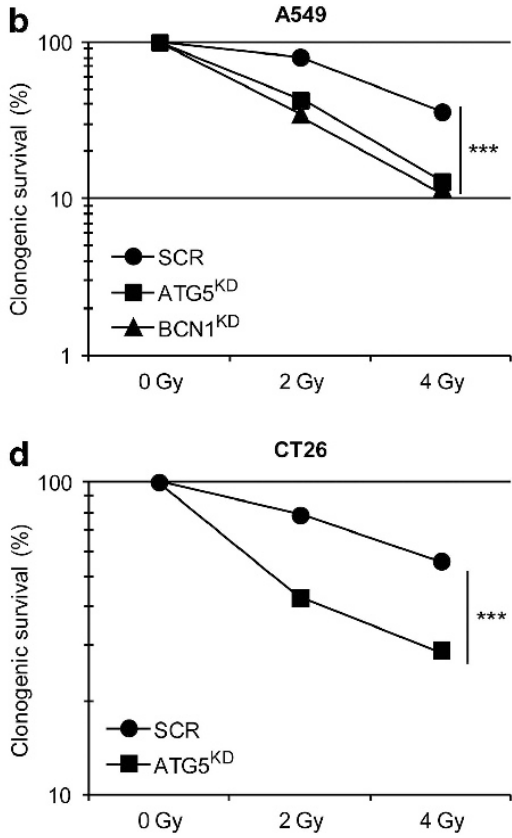

Figure 1 Knockdown of ATG5 or BECN 1 alters autophagy after irradiation and enhances radiation sensitivity of A549, H460 and CT26 cells. (a) Decreased ATG5 and BECN1 expression in A549 and $\mathrm{H} 460$ cells by RNA interference was observed by immunoblot. A representative immunoblot of three independent experiments is shown. (b-d) Cell lines as indicated. Radiation sensitivity of ATG5- and BECN1-depleted cells was assessed by clonogenic assay. Cells were transfected with a control shRNA or with an shRNA-targeting ATG5 or BECN1, then cells were left untreated or irradiated by 2 or 4 Gy. Cellular colonies were coloured and counted after 12-14 days and survival fraction (SF) was calculated and represented as described before. Quantitative data are reported as means \pm S.E.M. $\left(n=3\right.$ triplicate samples, Student's $t$-test, ${ }^{* * *} P<0.001$, as compared with untreated cells) 
procedure, we found that silencing of Beclin 1, yet another autophagy-relevant gene, in A549 and H460 cells significantly decreased the clonogenic survival following IR (Figures $1 \mathrm{~b}$ and $\mathrm{c}$ ), corroborating that autophagy indeed operates as a cytoprotective mechanism that counteracts irradiation-induced cell death.

ATG5 depletion induces cell death following IR. Given that autophagy inhibition reduced clonogenic survival following $I R$, we wondered whether cell death induction would be required for the reduction of clonogenic survival. To evaluate the contribution of different cell death modalities to this process, we simultaneously monitored the dissipation of the mitochondrial membrane potential $\left(\Psi_{m}\right)$ and the loss of plasma membrane integrity, which are indicative for apoptotic or necrotic events. We observed that IR of A549 cells with $4 \mathrm{~Gy}$ induced a decrease in $\Psi_{m}$ (as revealed by the loss of $\mathrm{DiOC}_{6}(3)$ staining $\left(\mathrm{DiOC}_{6}(3)^{\text {low }}\right)$ in the absence of plasma membrane permeabilization ( $\left.\mathrm{PI}^{\text {negative }}\right)$ indicating induction of lethal stress. The knockdown of ATG5, which abolishes autophagy (data not shown), led to a significant increase in the $\mathrm{Pl}^{-} \mathrm{DiOC}_{6}(3){ }^{\text {low }}$ population of cells after $6 \mathrm{~h}$ of irradiation with $4 \mathrm{~Gy}$ (Figures $2 \mathrm{a}$ and $\mathrm{b}$ ), indicating that the presence of ATG5 protects irradiated cells from death.

Depletion of ATG5 or Beclin 1 radiosensitizes human cancers xenografted on immuodeficient mice. Considering that IR-induced tumour cell death in vitro fails to explain some clinical observations in vivo, we decided to evaluate the effect of ATG5 and Beclin 1 depletion on the growth of irradiated A549 tumour xenografts implanted in immunodeficient BALB/C nude mice (which lack $T$ lymphocytes). As expected, control shRNA-transfected A549 tumours (SCR) presented a significant reduction in tumour size, as measured 24 days or 6 weeks after $8 \mathrm{~Gy}$ irradiation. This effect was significantly enhanced when autophagy-relevant genes ATG5 (Figure 3a) or Beclin 1 (Figure 3b) were knocked down. Then, we calculated the absolute growth delay (AGD) and the dose-enhancement factor (DEF) after irradiation to determine the impact of autophagy impairment on tumour growth after irradiation. AGD can be defined as the difference of time (determined in days) for non-irradiated versus irradiated tumours to reach a $50 \%$ volume increase. The DEF score was calculated as the difference in normalized tumour growth delay between irradiated autophagydeficient versus irradiated autophagy-proficient tumours. We found that AGD was 4, 49 and 39 days for control (SCR), ATG5-deficient $\left(\mathrm{ATG} 5^{\mathrm{KD}}\right.$ ) and BECN-1deficient $\left(\mathrm{BECN}-1^{\mathrm{KD}}\right)$ tumours, respectively. DEF was 4.5 for $\mathrm{ATG} 5^{\mathrm{KD}}$ tumours and 3.7 for $\mathrm{BECN}-1^{\mathrm{KD}}$ tumours, revealing ATG5 or BECN-1 depletion sensitized A549 tumours to IR.

Next, we evaluated the impact of irradiation-induced autophagy on the growth of xenografted H460 tumours. Similar to our previous results, control transfected $\mathrm{H} 460$ tumours (SCR) presented an average tumour volume of $293 \pm 29$ and $588 \pm 49 \mathrm{~mm}^{3}$ after 5 and 15 days of irradiation, respectively. In contrast, non-irradiated tumours revealed a mean tumour volume of $391 \pm 42$ and $1050 \pm 124 \mathrm{~mm}^{3}$ after 5 and 15 days of growth, respectively. ATG5-defective H460 $\left(\mathrm{ATG} 5^{\mathrm{KD}}\right.$ ) tumours were sensitized to irradiation and detected a significant enhancement in irradiation-induced tumour growth delay compared with autophagy-competent control tumours (Figure 3c). As compared with non-irradiated xenografts, the mean tumour volume of ATG5 ${ }^{\mathrm{KD}}$ tumours was significantly reduced 15 days after irradiation. Altogether, these results revealed that autophagy favours the growth of irradiated tumours in immunodeficient mice.

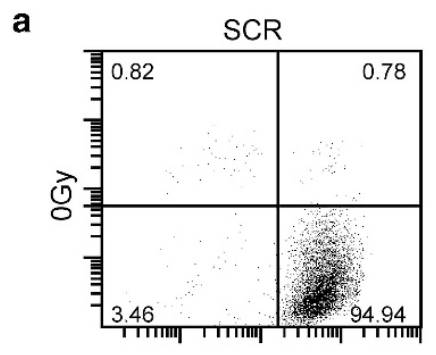

$\bar{\alpha}$
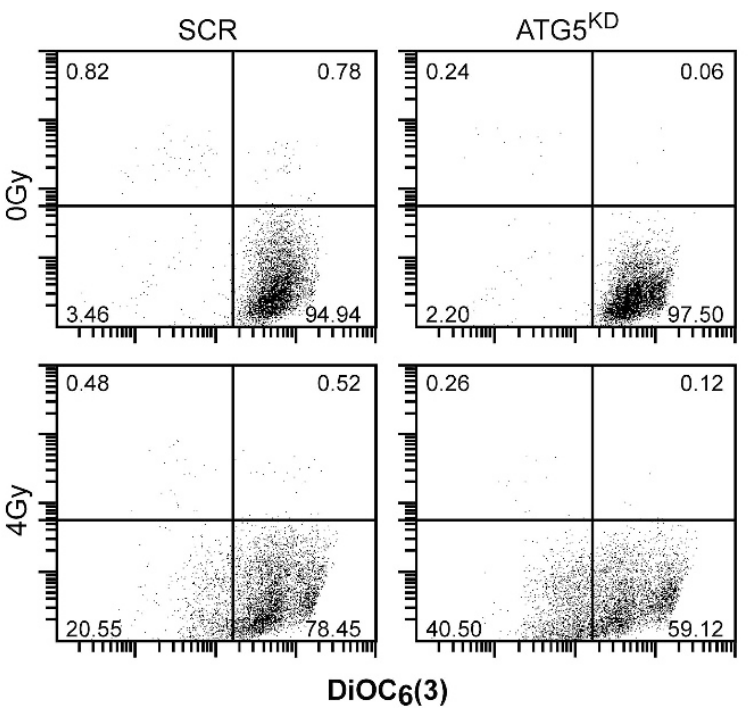

0.12

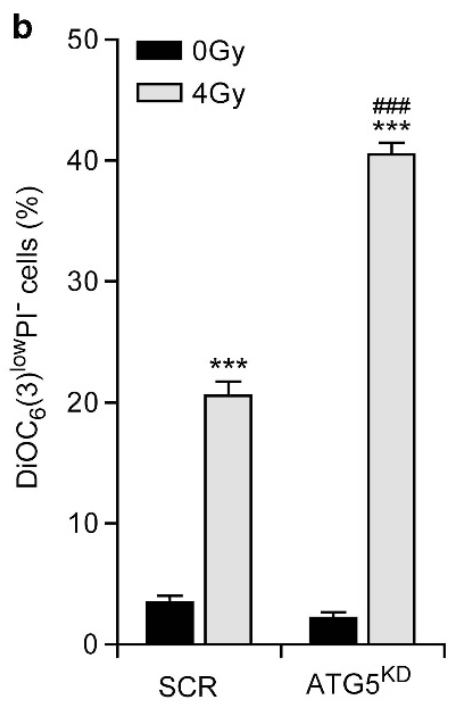

Figure 2 IR-induced cell death is increased in ATG5-depleted cells. (a) ATG5 depletion triggers mitochondrial membrane depolarization of A549 cells after ionizing radiation. A549 cells transfected by scrambled siRNA (SCR) or by specific shRNA for ATG5 (ATG5 ${ }^{\mathrm{KD}}$ ) were irradiated by 4 Gy and then subjected one day later to dual staining with the vital propidium iodide (PI) dye and with the mitochondrial transmembrane potential-sensitive dye 3,3 dihexyloxacarbocyanine iodide DiOC 6 (3) dye. Cell death was quantified by cytofluorometric analysis. (b) Histogram reporting the experiment shown in (a). Quantitative data are shown as means \pm S.E.M. ( $n=3$ triplicate samples, Student's $t$-test, ${ }^{* *} P<0.001$ comparing ATG5 ${ }^{\mathrm{KD}}$ (4 Gy) with ATG5 ${ }^{\mathrm{KD}}$ (0 Gy) and SCR (4 Gy) with SCR (0 Gy); ${ }^{\# \#} P<0.001$ comparing ATG5 ${ }^{\mathrm{KD}}$ (4 Gy) with SCR (4 Gy)) 

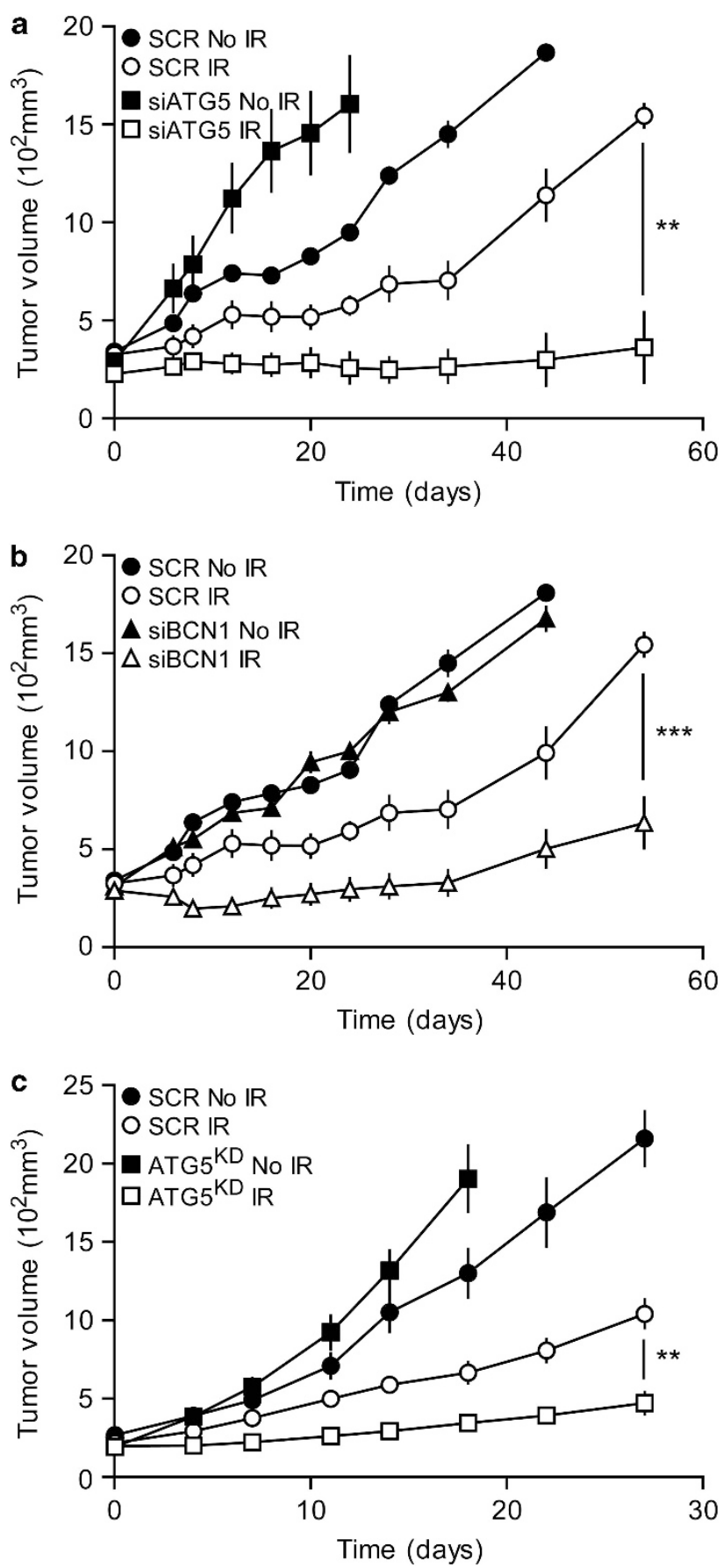

Figure 3 Autophagy delays radiation-induced growth in vivo. (a-c) Effects of ATG5 or BECN1 knockdowns on the growth of irradiated A549 or H460 tumours. Five nude BALB/c mice were subcutaneously injected with ATG5- or BECN1depleted A549 cells ( $\mathbf{a}$ and $\mathbf{b}$ ) or $\mathrm{H} 460$ cells (c). Once xenografted tumours became palpable, mice were randomly assigned to a control group or to a group that received localized tumour irradiation with a single dose $8 \mathrm{~Gy}$. Then, tumour volumes were monitored every $2-4$ days. Quantitative data are reported as means \pm S.E.M. (Student's t-test, ${ }^{\star \star} P<0.01,{ }^{{ }^{* \star}} P<0.001$, as compared with the control group)

ATG5 depletion impairs efficiency of tumour growth immunomodulation. Numerous preclinical studies suggest that the activation of the immune system is essential for successful radiotherapy. ${ }^{20-22}$ To evaluate the role of
IR-induced autophagy on anti-cancer immune responses, we analysed tumour growth of autophagy-deficient (ATG5 ${ }^{\mathrm{KD}}$ ) or -proficient (SCR) colon carcinoma CT26 cells (which are derived from BALB/c mice) in immunodeficient and immunocompetent BALB/c mice. Similar to our previous results with human tumour xenografts, knockdown of autophagy genes significantly enhanced the radiation-induced tumour growth delay for CT26-derived tumours growing on immunodeficient mice. Thus, in the context of an absent cellular immune response, ATG5-deficient $\left(A T G 5^{\mathrm{KD}}\right.$ ) tumours were significantly more sensitive to IR than autophagy-competent control tumours, as revealed by the comparison of tumour volumes measured before and after 10 days of irradiation (Figure 4a). In sharp contrast, autophagy inhibition resulted in a reduced tumour growth-inhibitory effect of radiotherapy, if autophagy-deficient or -competent cancers were implanted into immunocompetent BALB/c mice and then irradiated (Figure 4c). Taken together, these findings suggest that IRinduced autophagy has a major role in the onset of anticancer immune responses.

Following irradiation, the induction of immunogenic cell death (ICD) can lead to the emission of danger signals that are exposed at the cell surface or are released into the extracellular space, thus stimulating anti-tumour immune responses. ${ }^{23,24}$ Adenosine triphosphate (ATP) is one of the danger signals released during ICD. ${ }^{25,26}$ Considering that the onset of autophagy is associated with ATP release after chemotherapy, ${ }^{27}$ we decided to evaluate the effect of IRinduced autophagy on ATP release. Twenty-four hours post irradiation, autophagy-deficient $\left(\mathrm{ATG} 5^{\mathrm{KD}}\right.$ ) mouse colon carcinoma CT26 cells released significantly less ATP than their autophagy-competent counterparts (Figure 4e), demonstrating that IR-induced autophagy indeed modulates IR-induced ATP release. In line with previously published observations, ${ }^{27}$ administration of the ecto-ATPase inhibitor ARL67156 to CT26 cells increased the extracellular concentration of ATP after 4Gy irradiation in vitro (Figure 4e). In addition, intratumoural injection of ARL67156 increased the radiosensitivity of ATG5-deficient $\left(A T G 5^{\mathrm{KD}}\right.$ ) tumour cells in an immunocompetent context, indicating that it restored the immune response to irradiated ATG5 ${ }^{\mathrm{KD}}$ CT26 tumour cells (Figure 4d). The tumour growth-inhibitory effect of ARL67156 was lost in immunodeficient mice, indicating that it entirely relies on the presence of an intact immune system (Figure 4b).

Nine days post irradiation, autophagy-competent tumours were more heavily infiltrated by lymphocytes than autophagydeficient cancers, as revealed by histological analysis. Intratumoural injection of ARL67156 into ATG5-deficient $\left(A T G 5^{K D}\right.$ ) tumour cells significantly enhanced lymphocyte recruitment into irradiated ATG5 ${ }^{\mathrm{KD}}$ CT26 cancers (Figure 5), commensurate with the improved growth-inhibitory action of irradiation on such tumours.

\section{Discussion}

For decades, apoptosis has been considered as the principal cell death pathway elicited by radiotherapy, although numerous preclinical studies questioned this predominance. ${ }^{28}$ In vivo experiments have shown that mice lacking essential pro-apoptotic effectors (such as Bax and Bak, caspases, 
a

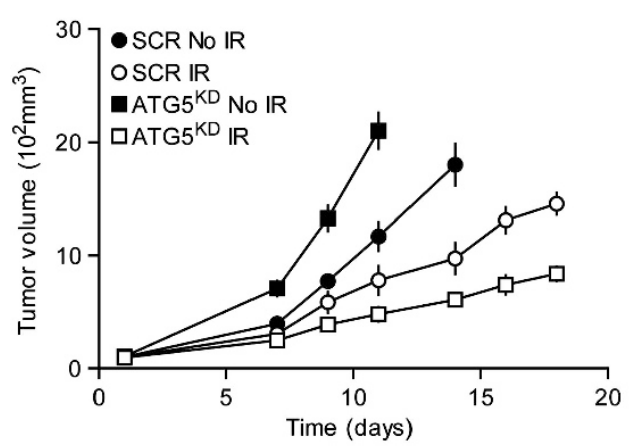

C

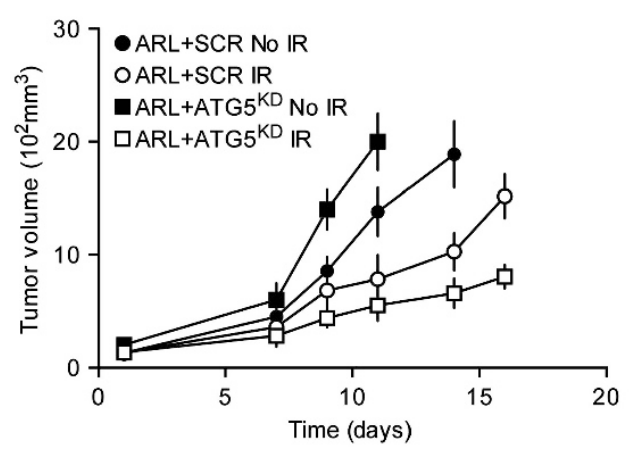

b

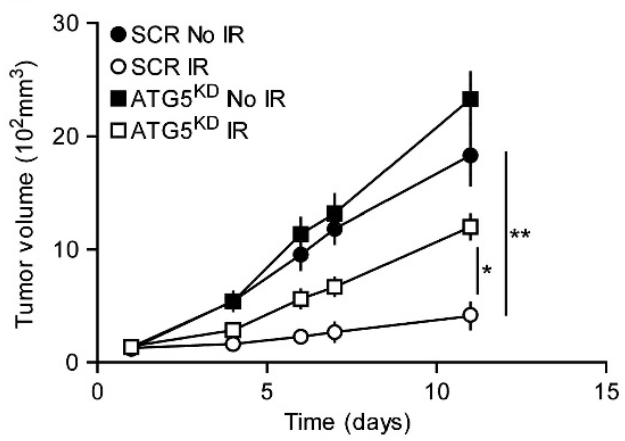

d

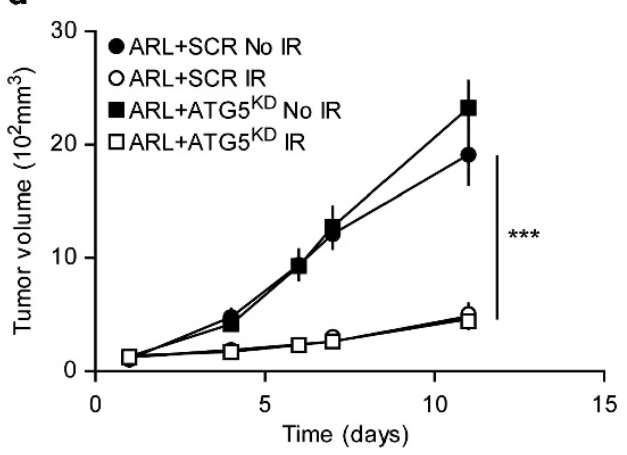

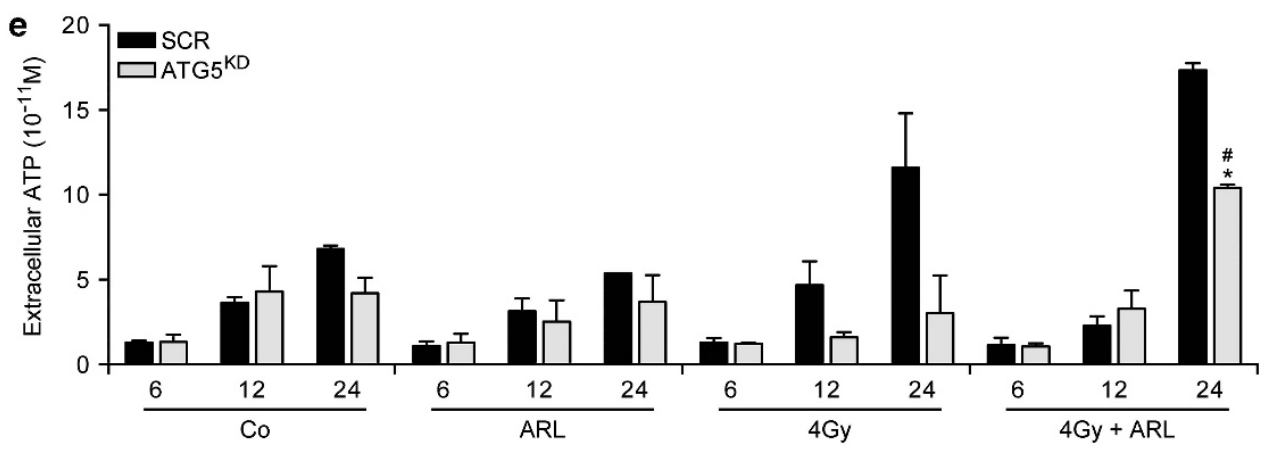

Figure 4 Autophagy inhibition decreases irradiation-induced ATP release and impairs anti-tumoural immune response. (a-c) Role of ATG5 on the growth of irradiated murine CT26 tumours implanted on immunodeficient mice. Nude BALB/c mice were subcutaneously injected with SCR or ATG5-depleted CT26 cells, then irradiated or left untreated with (c) or without an inhibitor of ecto-ATPase ARL67156 (ARL) (a). Tumour volumes were monitored every 3 or 4 days by direct measurement, as previously described. (b) Same experiment as in (a) in an immunocompetent host. Wild-type BALB/c mice were subcutaneously injected with ATG5-depleted CT26 cells, left untreated or irradiated, then tumour volume was monitored. Quantitative data presented are reported as means \pm S.E.M. (Student's $t$-test, ${ }^{*} P<0.05,{ }^{* *} P<0.01$ ). (d and e) Tumour growth of irradiated tumours is inhibited by ATP release via autophagy. To determine the impact of irradiation and autophagy on ATP release, ATG5depleted CT26 (ATG5 ${ }^{\mathrm{KD}}$ ) or control (SCR) cells were irradiated with 4 Gy and incubated with or without an inhibitor of ecto-ATPase ARL67156 (ARL). ATP release was determined in vitro by using enzymatic methods at indicated times (e). Quantitative data are reported as means \pm S.E.M. ( $n=3$ triplicate samples, Student's $t$-test, ${ }^{*} P<0.05$ comparing ATG ${ }^{\mathrm{KD}}$ (4 Gy + ARL) with ATG5 ${ }^{\mathrm{KD}}$ (Co) at $24 \mathrm{~h}$; ${ }^{*} P<0.05$ comparing ATG5 ${ }^{\mathrm{KD}}$ (4 Gy $+\mathrm{ARL}$ ) with ATG5 ${ }^{\mathrm{KD}}$ (4 Gy) at $24 \mathrm{~h}$ ). To assess the impact of extracellular ATP release by irradiation on tumour growth, Balb/c mice were injected with scramble or Atg5-depleted CT26 tumour cells, then xenografted tumours were randomly assigned to treatment groups that received localized tumour irradiation with one single $8 \mathrm{~Gy}$ irradiation, with ARL (d). Tumour volumes were determined as indicated above and quantitative data are reported as means \pm S.E.M. (Student's $t$-test, ${ }^{* \star} P<0.001$ )

Apaf-1 or caspase-9) exhibit relatively conserved phenotypes and do not completely suppress homeostatic programmed cell death. ${ }^{29}$ Non-apoptotic cell death morphologies or alternative death mechanisms have been discovered during radiation treatments, including 'autophagic cell death', programmed necrosis, mitotic catastrophe and entosis (also called cellular cannibalism). ${ }^{30,31}$ Recently, some preclinical results have highlighted the contribution of autophagy to radiation-induced cell death. ${ }^{15,32}$ Autophagy is a lysosomal degradation mechanism that is known to be essential to cellular homeostasis, as it is involved in degradation of misfolded proteins and removing dysfunctional organelles such as mitochondria. ${ }^{33}$ Thus, autophagy acts as a stress-responsive pathway that may confer protection against external stress to promote cell survival. ${ }^{34}$ Indeed, there is accumulating evidence that autophagy is upregulated in response to chemotherapy, radiation therapy or hypoxia. $^{35}$ 

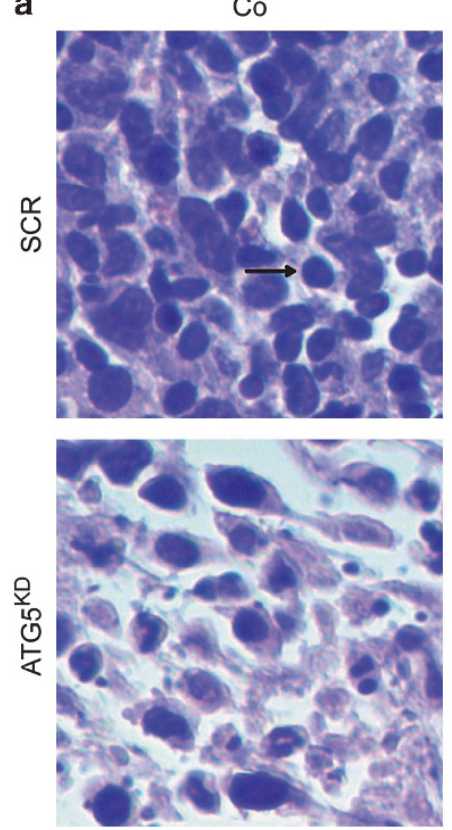

IR

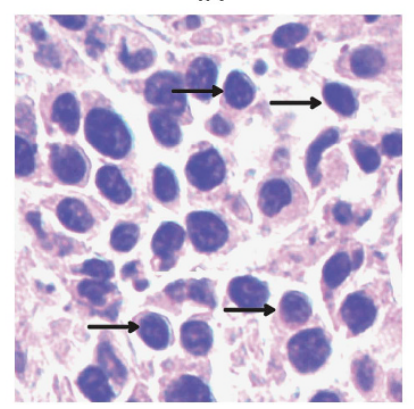

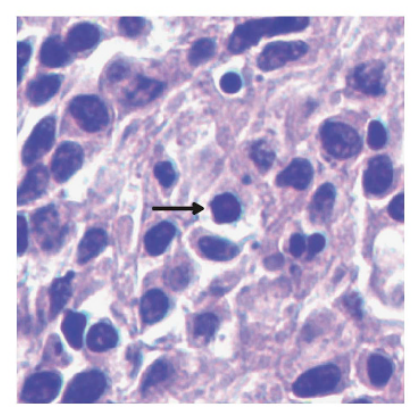

CR
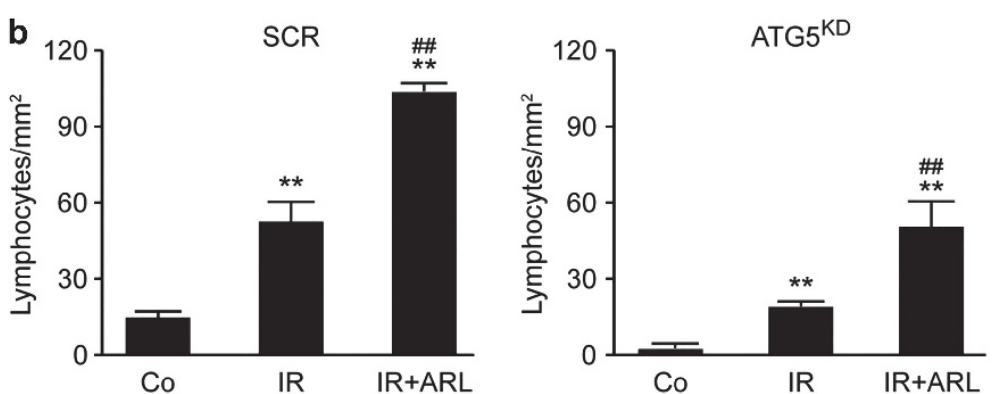

IR+ARL
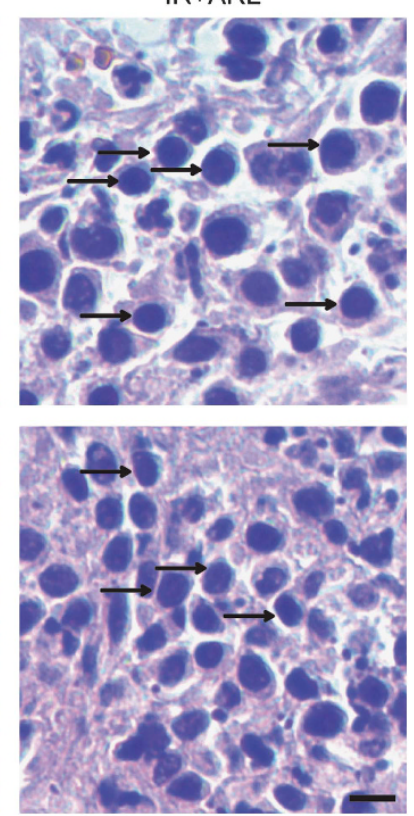

ATG $^{\mathrm{KD}}$

Figure 5 ARL67156 restores the lymphocyte infiltration in Atg5-depleted CT26 tumour after irradiation. (a) Representative pictures of CT26-recovered tumours. Scale bar, $10 \mu \mathrm{m}$. Arrows point lymphocytes. Quantitative data are reported in (b). Samples were compared using one-tailed Student's $t$-test. Error bars indicate S.E.M. ${ }^{*} P<0.01$, comparing SCR (IR) with SCR (Co) and SCR (IR + ARL) with SCR (Co); ${ }^{\# \#} P<0.01$, comparing SCR (IR + ARL) with SCR (IR); ${ }^{* *} P<0.01$ comparing ATG5 ${ }^{\mathrm{KD}}$ (IR) with $\mathrm{ATG}^{\mathrm{KD}}(\mathrm{Co})$ and $\mathrm{ATG5}{ }^{\mathrm{KD}}$ (IR $\left.+\mathrm{ARL}\right)$ with $\mathrm{ATG}^{\mathrm{KD}}(\mathrm{Co}) ;{ }^{\# \#} P<0.01$ comparing ATG5 ${ }^{\mathrm{KD}}$ (IR $\left.+\mathrm{ARL}\right)$ with ATG5 ${ }^{\mathrm{KD}}$ (IR) in (b)

The role of autophagy in radiation-induced cell death is barely understood. Some researchers suggested that simultaneous activation of apoptosis and autophagy enhances the sensitivity of radiation therapy both in vitro and in lung cancer xenograft models. ${ }^{17}$ In contrast, short-time inhibition of autophagy was reported to increase the cytotoxicity of radiotherapy in resistant cancer cells. ${ }^{15}$ On the basis of these results, we wondered whether combining autophagy inhibition with radiation therapy would enhance the efficiency of anti-tumour treatment. Clonogenic assays showed that knockdown of two different ATG (BECN1 and ATG5) had a radiosensitizing effect, suggesting that autophagy can prevent radiation-induced cell death. Consistent with data from other researchers, ${ }^{36-38}$ we found that autophagy inhibition enhanced tumour growth in vivo. However, as compared with autophagy-competent cancers, tumour cells with defective autophagy were significantly more sensitive to irradiation, in vivo, in an immunodeficient context.

The impact of inflammatory and immune responses on tumour progression is controversial. On one hand, chemokines secreted by tumours can promote tumour progression indirectly by stimulating leukocytes to secrete survival and neo-angiogenesis signals. ${ }^{39}$ On the other hand, dendritic cells have a major role in stimulating the anti-cancer immune response as they process tumour antigens and present them to other cells of the immune system. ${ }^{40}$ In specific circumstances, cancer cells can undergo immunogenic apoptosis, meaning that their corpses are engulfed by dendritic cells and their antigens are subsequently presented to tumour-specific $\mathrm{CD}^{+}{ }^{+} \mathrm{T}$ cells. ${ }^{41}$ Some successful chemotherapeutics induce immunogenic cell death, implying that the patient's dying cancer cells are converted into a therapeutic vaccine. Immunogenic cell death is characterized by the pre-apoptotic exposure of calreticulin (CRT) on the cell surface, post-apoptotic exodus of the chromatin-binding protein high-mobility group B1 (HMGB1) and release of ATP. Autophagy is not required for chemotherapy-induced CRT exposure and HMGB1 release, ${ }^{27}$ and our preliminary data suggest that autophagy is not required for radiationinduced CRT exposure or HMGB1 release either. Autophagy is strictly required for the release of ATP from dying tumour 
cells, ${ }^{27}$ which in turn is required for attracting immune cells including DC into the tumour. ${ }^{42}$ These results have been obtained in the context of immunogenic anti-neoplastic therapies. $^{27,42}$ Here, we report that, as compared with their autophagy-competent counterparts, autophagy-deficient cancer cells released reduced amounts of ATP also in the context of radiotherapy.

In this study, we wondered whether autophagy-dependent immunogenic signalling might affect the efficacy of radiotherapy. In experiments involving tumours implanted in immunodeficient nude mice, the radiosensitivity of $A T G 5^{K D}$. deficient cancers was higher than that of autophagy-competent controls. In stark contrast, however, autophagy-deficient tumours were less radiosensitive than control tumours in an immunocompetent context if the tumours evolved on BALB/C wild-type mice. The failure of IR-treated cancer cells depleted from Atg5 to induce a protective immune response in vivo could be corrected by intratumoural injections of ARL67156 (ARL), an inhibitor of ecto-ATPases (apyrases) that artificially increases extracellular ATP concentrations. ${ }^{43}$ ARL injections enhanced the efficacy of IR therapy against autophagydeficient CT26 cancers, hence significantly reducing tumour growth. As a control, ARL had no effect on autophagycompetent CT26 cancers. We conclude from these experiments that radiation-induced immunogenic cell death is associated with the autophagy-dependent extracellular accumulation of ATP.

In summary, this work underscores the important role of autophagy in the radiation response, both at the cell autonomous and at the immunological levels. To date, it is impossible to establish a link between autophagy and radiosensitivity without taking into account the immunological tumour microenvironment.

\footnotetext{
Materials and Methods

Cell lines. Human non-small-cell lung cancer cell lines A549 and H460, as well as mouse colon adenocarcinoma CT26 cells, were obtained from ATCC (Manassas, VA, USA). A549 cells were cultured in DMEM-F12 (Life Technologies, Carlsbad, CA, USA) medium containing $10 \%$ fetal bovine serum (FBS), $1 \%$ penicillin/streptomycin (PS) and $0.1 \%$ puromycin. $\mathrm{H} 460$ cancer cells were maintained in RPMI-1640 (Life Technologies) medium containing 10\% FBS and 1\% PS. CT26 cells were cultured in RPMI-1640 medium (Life Technologies) containing 10\% FBS, 1\% PS and $0.1 \%$ puromycin. All cells were maintained under $5 \% \mathrm{CO}_{2}$ humidified atmosphere at $37^{\circ} \mathrm{C}$.
}

RNA interference. To generate A549 and $\mathrm{H} 460$ cells that stably express short hairpin RNA (shRNA) targeting ATG5 and BECN1, the corresponding constructs (obtained from Origene, Rockville, MD, USA) were transfected together with scrambled control vectors into retrovirus producing Phoenix ampho cells by means of Lipofectamin 2000 (Life Technologies) following the manufacturer's instructions. ShRNA-encoding viruses were used to infect A549 cells in the presence of $4 \mu \mathrm{g} / \mathrm{ml}$ polybrene (Millipore, Billerica, MA, USA). Cells transduced with shRNA were selected in $3 \mu \mathrm{g} / \mathrm{ml}$ puromycin (InvivoGen, San Diego, CA, USA) and single clones were obtained by limiting dilution. Insertion was validated by immunoblotting with the suitable antibodies. For the generation of autophagydeficient CT26, a set of plasmids encoding shRNAs specific for murine Atg5 plus a control shRNA were obtained from Origen and used as described above.

Small interfering RNA control (UNR, universal negative control \#1) or specific for human ATG5 (sense $5^{\prime}$-CCUUUGGCCUAAGAAGAAATTdTdT-3') or for human BECN1 (sense $5^{\prime}$-CAGUGGAUCUAAAUCUCAATTdTdT-3') were purchased from Sigma-Proligo (The Woodlands, TX, USA). H460 and A549 cells were transfected (at $30-40 \%$ confluence) with the HiPerFect transfection reagent (Qiagen, Hilden, Germany)-previously complexed with $100 \mathrm{nM}$ siRNA-as instructed by the manufacturer. Transfected cells were used for experiments no earlier than $48 \mathrm{~h}$ after transfection. Protein knockdown was confirmed by immunoblotting.

Western blot analysis. A549 or $\mathrm{H} 460$ cells were washed with PBS, then exposed to cell lysis buffer containing $100 \mathrm{mM} \mathrm{NaCl}, 50 \mathrm{mM}$ Tris- $\mathrm{HCl}$ pH 7.4, $5 \mathrm{mM}$ EDTA pH8, $50 \mathrm{mM} \mathrm{NaF}, 1 \%$ IGEPAL CA-630 (Sigma-Aldrich, St. Louis, MO, USA), $1 \mathrm{mM} \mathrm{NaOV}$ (phosphatase inhibitor), 1X protease inhibitor (Boehringer, Ingelheim-Am-Rhein, Germany). After protein extraction on ice, protein dosage was performed using the Bio-Rad Protein Assay Kit (Bio-Rad, Hercules, MA, USA) as recommended by the manufacturer, then the protein concentration was measured using the Bio-Rad microplate reader Model 680 system equipped with Microplate manager 5.2 software (Bio-Rad). Following denaturing at $95^{\circ} \mathrm{C}$ for $5 \mathrm{~min}, 30 \mu \mathrm{g}$ of proteins were loaded onto denaturing acrylamide gel at $4-12 \%$ (NuPaAGE Bis-tris Gel, Life Technologies). Migration was performed at $200 \mathrm{~V}$ for $90 \mathrm{~min}$. Proteins were blotted onto nitrocellulose by electro-transfer at $40 \mathrm{~V}$ for $1 \mathrm{~h}$. Membranes were washed with TBS containing at $0.1 \%$ Tween-20 (TBS-T) and then blocked for $1 \mathrm{~h}$ in TBS-T containing $5 \%$ bovine serum albumin. The membranes were incubated overnight at $4^{\circ} \mathrm{C}$ with the following primary antibodies: anti-ATG 5 (from Cell Signaling Technologies, Danvers, MA, USA) and anti-BECN1 (from Santa Cruz, CA, USA). Horseradish peroxidase-labelled goat anti-rabbit or anti-mouse antibodies from GE Healthcare (Buckinghamshire, UK) were used for signal detection with direct scanning (Image Quant 350, GE Healthcare). Measurements were normalized by assessing expression of glyceraldehyde-3-phosphate dehydrogenase (GAPDH, from Biodesign International, Saco, ME, USA).

Clonogenic survival and combination studies. Transfected and untransfected cells were plated at different cellular density. The day after, cells were irradiated at doses ranging from 0 to $4 \mathrm{~Gy}$ (Cesium $=\mathrm{Cs}^{137}, 1 \mathrm{~Gy} / \mathrm{min}$, gamma irradiator IBL-637 from CIS-Biolnternational, IBA, Saclay, France). Then, cells were incubated for 10-12 days under standard culture conditions and thereafter, cellular colonies were coloured with crystal violet, washed and counted. Only cellular colonies with more than 50 cells were taken into consideration. Each surviving fraction (SF) was calculated by dividing the number of cellular clones by the number of cells plated and was further normalized based on the ratio of clonogenic survival for each radiation dose and clonogenic survival of non-irradiated controls. The sensitivity enhancement ratio for each radiation dose was calculated by dividing the SF of transfected cells by the SF of untransfected cells.

Cell death detection. Scrambled and ATG5 shRNA expressing cells were irradiated $24 \mathrm{~h}$ after plating. After $6 \mathrm{~h}$, cell death was quantified by cytofluorometric analysis using a FACS Vantage (Becton Dickinson, Mountain View, CA, USA). Thus, cells were stained with $40 \mathrm{nM} 3,3$ dihexyloxacarbocyanine iodide $\left(\operatorname{DiOC}_{6}(3)\right.$; Molecular Probes, Eugene, OR, USA) for $30 \mathrm{~min}$ at $37^{\circ} \mathrm{C}$ and concomitantly with $1 \mu \mathrm{g} / \mathrm{ml}$ propidium iodide (PI; Sigma-Aldrich) for $30 \mathrm{~min}$ at $37^{\circ} \mathrm{C}$ to determine the mitochondrial transmembrane potential. Data were statistically evaluated using CellQuest Pro software (BD Biosciences, San Diego, CA, USA).

In vivo assessments. $\mathrm{A} 549, \mathrm{H} 460$ and $\mathrm{CT} 26$ cells stably transfected with a shRNA-targeting ATG5 or BECN1 were grown as tumour xenografts in the right flank of nude or BALB/c mice aged from 6 to 8 weeks from Janvier CERT (Le Genest, St. Isle, France). Tumour-bearing mice were randomly assigned to treatment groups (at least six in each group) and mice from the irradiation group received localized tumour irradiation with single dose IR (8Gy) using $225 \mathrm{kV}$ COMET MRX-225/22 X-ray (Stamford, CT, USA) at a dose rate of $0.35 \mathrm{~Gy} / \mathrm{min}(200 \mathrm{kV}, 15 \mathrm{~mA}, 0.2 \mathrm{~mm} \mathrm{Cu}$ filter). Control groups received no irradiation. Tumour size and body weight were measured twice a week. Tumour volume was calculated using the formula: (length $\times$ width $\left.^{2}\right) / 2$. Mice were sacrificed when the tumour volume reached $1500-2000 \mathrm{~mm}^{3}$ (Figures 3 and 4) or at day 9 after IR (Figure 5).

Animal experimentations. Animals were maintained in specific pathogenfree conditions, and experiments followed the Federation of European Laboratory Animal Science Association (FELASA) guidelines. Animal experiments were approved by the local Ethics Committee (CEEA IRCIV / IGR n 26, registered with the French Ministry of Research) and were in compliance with EU 63/2010 directive. Animals were used between 6 and 20 weeks of age and those bearing tumours exceeding $20-25 \%$ body mass were killed. BALB/c $(\mathrm{H}-2 \mathrm{~d})$ mice were 
obtained from Janvier (Le Genest). The in vivo experiments were carried out under the animal care licence no. D94-076-11 (Ministère de l'Agriculture).

ATP release assays in vitro. Extracellular ATP levels were measured by the luciferin-based ENLITEN ATP Assay (Promega, Fitchburg, WI, USA) and ATP assay (Calbiochem, Nottingham, UK) kits, respectively, in excess of luciferin and luciferase, as indicated by the manufacturer

Histology and HES staining. Recovered tumours were fixed with 4\% PFA for $3 \mathrm{~h}$ and then embedded into paraffin. Sections of $4 \mu \mathrm{m}$ paraffin were fixed and stained with haematoxylin and eosin according to standard protocols.

\section{Conflict of Interest}

The authors declare no conflict of interest.

Acknowledgements. This work was supported by a grant from Association de recherche contre le cancer (ARC) and Électricité de France (EDF) to ED. We thank Fondation Gustave Roussy. We also thank Didier Métivier (INSERM U848, Villejuif, France) for technical help and Nazanine Modjtahedi (INSERM U848, Villejuif, France) for helpful advice. This work is supported by grants to GK from the Ligue Nationale contre le Cancer (Equipe labellisée), Agence Nationale pour la Recherche (ANR), Fondation pour la Recherche Médicale (FRM), Institut National du Cancer (INCa), Cancéropôle lle-de-France, Fondation Bettencourt-Schueller, the LabEx Onco-Immunology and the Paris Alliance of Cancer Research Institutes.

1. Gudkov AV, Komarova EA. The role of p53 in determining sensitivity to radiotherapy. Nat Rev Cancer 2003; 3: 117-129.

2. Eriksson D, Stigbrand T. Radiation-induced cell death mechanisms. Tumour Biol 2010; 31 363-372.

3. Paglin S, Hollister T, Delohery T, Hackett N, McMahill M, Sphicas E et al. A novel response of cancer cells to radiation involves autophagy and formation of acidic vesicles. Cancer Res 2001; 61: 439-444.

4. Stewart RD, Yu VK, Georgakilas AG, Koumenis C, Park JH, Carlson DJ. Effects of radiation quality and oxygen on clustered DNA lesions and cell death. Radiat Res 2011 176: $587-602$.

5. Mizushima N, Komatsu M. Autophagy: renovation of cells and tissues. Cell 2011; 147 728-741.

6. Marino G, Martins I, Kroemer G. Autophagy in Ras-induced malignant transformation: fatal or vital? Mol Cell 2011; 42: 1-3.

7. Mizushima N. The pleiotropic role of autophagy: from protein metabolism to bactericide. Cell Death Differ 2005; 12(Suppl 2): 1535-1541.

8. Klionsky DJ, Cregg JM, Dunn WA Jr. Emr SD, Sakai Y, Sandoval IV et al. A unified nomenclature for yeast autophagy-related genes. Dev Cell 2003; 5: 539-545.

9. Klionsky DJ, Emr SD. Autophagy as a regulated pathway of cellular degradation. Science 2000; 290: 1717-1721.

10. Ohsumi Y. Molecular dissection of autophagy: two ubiquitin-like systems. Nat Rev Mol Cell Biol 2001; 2: 211-216.

11. Petiot A, Ogier-Denis E, Blommaart EF, Meijer AJ, Codogno P. Distinct classes of phosphatidylinositol 3'-kinases are involved in signaling pathways that control macroautophagy in HT-29 cells. J Biol Chem 2000; 275: 992-998.

12. Yang Z, Klionsky DJ. Eaten alive: a history of macroautophagy. Nat Cell Biol 2010; 12 : 814-822.

13. Rabinowitz JD, White E. Autophagy and metabolism. Science 2010; 330: 1344-1348.

14. Ito H, Daido S, Kanzawa T, Kondo S, Kondo Y. Radiation-induced autophagy is associated with LC3 and its inhibition sensitizes malignant glioma cells. Int J Oncol 2005; 26 : $1401-1410$.

15. Apel A, Herr I, Schwarz H, Rodemann HP, Mayer A. Blocked autophagy sensitizes resistant carcinoma cells to radiation therapy. Cancer Res 2008; 68: 1485-1494.

16. Chen YS, Song HX, Lu Y, Li X, Chen T, Zhang Y et al. Autophagy inhibition contributes to radiation sensitization of esophageal squamous carcinoma cells. Dis Esophagus 2011; 24 437-443

17. Kim KW, Moretti L, Mitchell LR, Jung DK, Lu B. Combined Bcl-2/mammalian target of rapamycin inhibition leads to enhanced radiosensitization via induction of apoptosis and autophagy in non-small cell lung tumor xenograft model. Clin Cancer Res 2009; 15 . 6096-6105.
18. Lin $\mathrm{Cl}$, Whang EE, Abramson MA, Jiang X, Price BD, Donner DB et al. Autophagy: a new target for advanced papillary thyroid cancer therapy. Surgery 2009; 146 : 1208-1214.

19. Lomonaco SL, Finniss S, Xiang C, Decarvalho A, Umansky F, Kalkanis SN et al. The induction of autophagy by gamma-radiation contributes to the radioresistance of glioma stem cells. Int J Cancer 2009; 125: 717-722.

20. Stone HB, Peters LJ, Milas L. Effect of host immune capability on radiocurability and subsequent transplantability of a murine fibrosarcoma. J Natl Cancer Inst 1979; 63: $1229-1235$

21. Apetoh L, Ghiringhelli F, Tesniere A, Obeid M, Ortiz C, Criollo A et al. Toll-like receptor 4-dependent contribution of the immune system to anticancer chemotherapy and radiotherapy. Nat Med 2007; 13: 1050-1059.

22. Ladoire S, Hannani D, Vetizou M, Locher C, Aymeric L, Apetoh L et al. Cell death associated molecular patterns (CDAMP) as determinants of cancer immunogenicity. Antiox Redox Signal 2013; e-pub ahead of print 8 February 2013; PubMed PMID: 23394620

23. Obeid M, Panaretakis T, Joza N, Tufi R, Tesniere A, van Endert P et al. Calreticulin exposure is required for the immunogenicity of gamma-irradiation and UVC light-induced apoptosis. Cell Death Differ 2007; 14: 1848-1850.

24. Obeid M, Tesniere A, Panaretakis T, Tufi R, Joza N, van Endert $P$ et al. Ecto-calreticulin in immunogenic chemotherapy. Immunol Rev 2007; 220: 22-34.

25. Elliott MR, Chekeni FB, Trampont PC, Lazarowski ER, Kadl A, Walk SF et al. Nucleotides released by apoptotic cells act as a find-me signal to promote phagocytic clearance. Nature 2009; 461: 282-286.

26. Tsukimoto M, Homma T, Ohshima Y, Kojima S. Involvement of purinergic signaling in cellular response to gamma radiation. Radiat Res 2010; 173: 298-309.

27. Michaud M, Martins I, Sukkurwala AQ, Adjemian S, Ma Y, Pellegatti P et al. Autophagydependent anticancer immune responses induced by chemotherapeutic agents in mice. Science 2011; 334: 1573-1577.

28. Haas-Kogan DA, Dazin P, Hu L, Deen DF, Israel A. P53-independent apoptosis: a mechanism of radiation-induced cell death of glioblastoma cells. Cancer J Sci Am 1996; 2: $114-121$.

29. Chautan M, Chazal G, Cecconi F, Gruss P, Golstein P. Interdigital cell death can occur through a necrotic and caspase-independent pathway. Curr Biol 1999; 9: 967-970.

30. Yuan J, Kroemer G. Alternative cell death mechanisms in development and beyond. Genes Dev 2010: 24: 2592-2602.

31. Galluzzi L, Vitale I, Abrams JM, Alnemri ES, Baehrecke EH, Blagosklonny MV et al. Molecular definitions of cell death subroutines: recommendations of the Nomenclature Committee on Cell Death 2012. Cell Death Differ 2012; 19: 107-120.

32. Liang B, Kong D, Liu Y, Liang N, He M, Ma S et al. Autophagy inhibition plays the synergetic killing roles with radiation in the multi-drug resistant SKVCR ovarian cancer cells. Radiat Oncol 2012; 7: 213

33. Wang K, Klionsky DJ. Mitochondria removal by autophagy. Autophagy 2011; 7: 297-300.

34. White E, DiPaola RS. The double-edged sword of autophagy modulation in cancer. Clin Cancer Res 2009; 15: 5308-5316.

35. Mancias JD, Kimmelman AC. Targeting autophagy addiction in cancer. Oncotarget 2011; 2: $1302-1306$

36. Mathew R, Karp CM, Beaudoin B, Vuong N, Chen G, Chen HY et al. Autophagy suppresses tumorigenesis through elimination of p62. Cell 2009; 137: 1062-1075.

37. Qu X, Yu J, Bhagat G. Furuya N, Hibshoosh H, Troxel A et al. Promotion of tumorigenesis by heterozygous disruption of the beclin 1 autophagy gene. J Clin Invest 2003; 112: 1809-1820.

38. Marino G, Salvador-Montoliu N, Fueyo A, Knecht E, Mizushima N, Lopez-Otin C Tissue-specific autophagy alterations and increased tumorigenesis in mice deficient in Atg4C/autophagin-3. J Biol Chem 2007; 282: 18573-18583.

39. Raman D, Baugher PJ, Thu YM, Richmond A. Role of chemokines in tumor growth. Cancer Lett 2007; 256: 137-165

40. Mantovani A, Romero P, Palucka AK, Marincola FM. Tumour immunity: effector response to tumour and role of the microenvironment. Lancet 2008; 371: 771-783.

41. Zitvogel L, Apetoh L, Ghiringhelli F, Kroemer G. Immunological aspects of cancer chemotherapy. Nat Rev Immunol 2008; 8: 59-73.

42. Saitoh T, Akira S. Regulation of innate immune responses by autophagy-related proteins. J Cell Biol 2010; 189: 925-935

43. Mandapathil M, Hilldorfer B, Szczepanski MJ, Czystowska M, Szajnik M, Ren J et al. Generation and accumulation of immunosuppressive adenosine by human CD4 + CD25highFOXP3 + regulatory T cells. J Biol Chem 2010; 285: 7176-7186.

(i) This work is licensed under a Creative Commons Attribution 3.0 Unported License. To view a copy of this license, visit http://creativecommons.org/licenses/by/3.0/ 